\title{
Formulas for $\mathrm{TE}_{01}$ cutoff in optical fibers with arbitrary index profile
}

\author{
Yasuo Kokubun and Kenichi Iga \\ Tokyo Institute of Technology, Research Laboratory of Precision Machinery and Electronics, 4259 Nagatsuta, Midori-ku, Yokohama, \\ 227 Japan \\ (Received 7 May 1979)
}

\begin{abstract}
The cutoff problem of TE modes of a radially inhomogeneous optical fiber is transformed into an integral equation by using a Sturm-Liouville-type boundary problem. Three types of approximate formulas for calculating the cutoff frequency of the $\mathrm{TE}_{01}$ mode are derived by calculating the eigenvalue of this integral equation. The comparison of the cutoff frequencies calculated by means of these formulas with some exact values indicates that the third approximate formula gives the best accuracy (within $10^{-3} \%$ ) and requires only a few seconds of computation time. The effect of the center dip on the cutoff frequency is investigated. The single-mode condition of single-mode fibers with any arbitrary refractive index profile can be obtained with high accuracy.
\end{abstract}

\section{INTRODUCTION}

The single-mode fiber is promising as a long distance broadband information transmission medium. Furthermore, the enlargement of its core radius ${ }^{1}$ and wavelength multiplexing ${ }^{2}$ utilizing the dual-mode region have been proposed. In order to design these single-mode and dual-mode fibers, it is necessary to grasp precisely the cutoff condition of the $\mathrm{TE}_{01}, \mathrm{TM}_{01}$, and $\mathrm{HE}_{21}$ modes. Especially, the core radius of a drawn fiber required for single-mode transmission can be obtained in the stage of a preform rod, when the refractive index profile of the preform rod is measured and the refractive index profile changes very little while drawing the fiber. Therefore, a formula for calculating the single-mode condition from the refractive index profile becomes necessary. However, the single-mode condition has been difficult to calculate analytically with a few exceptions of step-index, ${ }^{3}$ parabolicindex, ${ }^{4}$ and $W$-type fibers, ${ }^{5,6}$ and so we are compelled to calculate it numerically. Thus far, as the approximate formula for calculating the single-mode condition, the power-series expansion $\operatorname{method}^{7}$ and variational method ${ }^{8}$ have been proposed. But the former method can be applied only to profiles expressed by an $\alpha$-power law and power-series expansion, and the latter method is not always of satisfactory accuracy (the error is more than $1 \%){ }^{8}$

In this paper, (i) the cutoff problem of TE modes in a radially inhomogeneous optical fiber is transformed into an integral equation by utilizing the fact that both the wave equation and the characteristic equation at cutoff constitute a Sturm-Liouville-type boundary problem; and, (ii) several types of approximate formulas for calculating the cutoff frequency of the $\mathrm{TE}_{01}$ mode are derived by using approximate formulas for the eigenvalue of an integral equation. Cutoff frequencies of some index profiles are calculated by means of these formulas and the accuracy is investigated.

\section{CUTOFF CONDITION OF TE MODES}

Suppose the radially inhomogeneous optical fiber whose refractive index is given as a function of transverse distance $\rho$ normalized by the core radius $a$ by

$$
\begin{aligned}
n^{2}(\rho) & =n_{1}^{2}[1-2 \Delta f(\rho)] & & \rho \leqq 1 \\
& =n_{2}^{2} & & \rho>1
\end{aligned}
$$

where $2 \Delta=\left(n_{1}^{2}-n_{2}^{2}\right) / n_{1}^{2}, n_{1}$ is the maximum value of the refractive index, and so $f(\rho) \geqq 0$. The scalar wave equation and the characteristic equation are given by

$$
\begin{gathered}
\frac{1}{\rho} \frac{d}{d \rho}\left(\rho \frac{d R(\rho)}{d \rho}\right)+\left\{V^{2}[1-b-f(\rho)]-\frac{1}{\rho^{2}}\right\} R(\rho)=0, \\
R(0)=0 \\
{\left[\frac{1}{R(\rho)} \frac{d R(\rho)}{d \rho}\right]_{\rho=1}=-\frac{w K_{0}(w)}{K_{1}(w)}-1,}
\end{gathered}
$$

where $K_{n}(x)$ is the modified Bessel function of the second kind. The parameters $V, b$, and $w$ are defined by

$$
\begin{gathered}
V=k_{0} n_{1} a \sqrt{2 \Delta} \quad\left(k_{0}=2 \pi / \lambda\right), \\
b=\frac{\left(\beta / k_{0}\right)^{2}-n_{2}^{2}}{n_{1}^{2}-n_{2}^{2}}, \\
w=\sqrt{\beta^{2}-k_{0}^{2} n_{2}^{2}}=V \sqrt{b},
\end{gathered}
$$

where $\beta$ is the propagation constant and $k_{0}=2 \pi / \lambda$. Since Eqs. (2), (3), and (4) constitute a Sturm-Liouville-type boundary problem, these conditions can be transformed into an integral equation in terms of a Green's function as follows:

$$
R(t)=V^{2} \int_{0}^{1} G(t, s) s[1-f(s)] R(s) d s .
$$

The Green's function in the case of $b \neq 0(w \neq 0)$ is given by

$$
G(t, s)= \begin{cases}I_{1}(w s) K_{1}(w t) & 0 \leqq s<t \leqq 1 \\ I_{1}(w t) K_{1}(w s) & 0 \leqq t<s \leqq 1\end{cases}
$$

where $I_{n}(x)$ and $K_{n}(x)$ are the modified Bessel functions of the first and second kinds, respectively.

At cutoff, $\beta=n_{2} k$ and $b$ reaches zero ( $w$ also reaches zero). In the limit of $b \rightarrow 0$ and $w \rightarrow 0$, Eqs. (2) and (4) become

$$
\begin{gathered}
\frac{1}{\rho} \frac{d}{d \rho}\left(\rho \frac{d R(\rho)}{d \rho}\right)+\left\{V_{c}^{2}[1-f(\rho)]-\frac{1}{\rho^{2}}\right\} R(\rho)=0, \\
{\left[\frac{1}{R(\rho)} \frac{d R(\rho)}{d \rho}\right]_{\rho=1}=-1 .}
\end{gathered}
$$

The condition Eq. (3) does not change. Equations (10), (3), and (11) also constitue a Sturm-Liouville-type boundary problem and so these are transformed into the following integral equation: 


$$
R(t)=V_{c}^{2} \int_{0}^{1} G_{c}(t, s) s[1-f(s)] R(s) d s,
$$

where the Green's function $G_{c}(t, s)$ at cutoff is given by

$$
G_{c}(t, s)= \begin{cases}\frac{1}{2} \frac{s}{t} & 0 \leqq s<t \leqq 1 \\ \frac{1}{2} \frac{t}{s} & 0 \leqq t<s \leqq 1 .\end{cases}
$$

The cutoff frequency $V_{c}$ of the $\mathrm{TE}_{01}$ mode is obtained as the minimum eigenvalue of Eq. (8b).

\section{APPROXIMATE FORMULA FOR CALCULATING THE MINIMUM EIGENVALUE}

By multiplying both sides of Eq. (8b) by $\sqrt{t[1-f(t)]}$ and using the following transformations,

$$
\begin{aligned}
& y(t)=\sqrt{t[1-f(t)]} R(t), \\
& K(t, s)= \begin{cases}\frac{1}{2} \sqrt{t[1-f(t)]} \frac{s}{t} \sqrt{s[1-f(s)]} & 0 \leqq s<t \leqq 1 \\
\frac{1}{2} \sqrt{t[1-f(t)]} \frac{t}{s} \sqrt{s[1-f(s)]} & 0 \leqq t<s \leqq 1,\end{cases}
\end{aligned}
$$

the following symmetric kernel integral equation is obtained:

$$
y(t)=V_{c}^{2} \int_{0}^{1} K(t, s) y(s) d \dot{s} .
$$

\section{A. Approximate solution by means of the iterated kernel}

Suppose a general symmetric kernel integral equation

$$
y(t)=\lambda \int_{a}^{b} K(t, s) y(s) d s,
$$

whose eigenvalues are $\lambda_{1}, \lambda_{2}, \ldots, \lambda_{\mathrm{i}}, \ldots$ in increasing order of magnitude, and their corresponding eigenfunctions are designated by $\psi_{1}(t), \psi_{2}(t), \ldots, \psi_{i}(t), \ldots$, respectively. Let us define the iterated kernel of the symmetric kernel $K(t, s)$ by

$$
\begin{gathered}
K^{2}(t, s)=\int_{0}^{1} K(t, \xi) K(\xi, s) d \xi \\
\vdots \\
K^{n}(t, s)=\int_{0}^{1} K(t, \xi) K^{n-1}(\xi, s) d \xi .
\end{gathered}
$$

Then the $n$ th-order iterated kernel can be expanded in terms of the orthogonal sets ${ }^{9}$ of $\psi_{i}(t)$ and $\psi_{i}(s)$ as

$$
K^{n}(t, s)=\sum_{i=1}^{\infty} \frac{\psi_{i}(t) \psi_{i}(s)}{\lambda_{i}^{n}} .
$$

Because of the orthonormality of eigenfunctions $\psi_{i}(t)$ and $\psi_{i}(s)(i=1,2, \ldots)$, the following equation is obtained:

$$
T_{2 m} \equiv \int_{a}^{b} K^{2 m}(t, t) d t=\sum_{i=1}^{\infty}\left(\frac{1}{\lambda_{i}^{2}}\right)^{m} .
$$

Since the inequalities $\lambda_{1}<\lambda_{2}<\ldots<\lambda_{i}<\ldots$ hold, the minimum eigenvalue $\lambda_{1}$ can be approximated by

$$
\lambda_{1} \simeq \frac{1}{\left(T_{2 m}\right)^{1 / 2 m}} .
$$

Now, putting $\lambda_{1}=V_{c}^{2}, a=0$, and $b=1$, the iterated kernel of Eq. (14) can be calculated by means of Eq. (19) as follows:

$$
K^{2}(t, s)=\left\{\begin{array}{l}
\frac{1}{4} \sqrt{t[1-f(t)]} \sqrt{s[1-f(s)]} \\
\times\left[\frac{1}{t s} \int_{0}^{s} \xi^{3}[1-f(\xi)] d \xi\right. \\
+\frac{s}{t} \int_{s}^{t} \xi[1-f(\xi)] d \xi \\
\left.+t s \int_{t}^{1} \frac{1}{\xi}[1-f(\xi)] d \xi\right] 0 \leqq s<t \leqq 1, \\
\frac{1}{4} \sqrt{t[1-f(t)]} \sqrt{s[1-f(s)]} \\
\times\left[\frac{1}{t s} \int_{0}^{t} \xi^{3}[1-f(\xi)] d \xi\right. \\
+\frac{t}{s} \int_{t}^{s} \xi[1-f(\xi)] d \xi \\
\left.+t s \int_{s}^{1} \frac{1}{\xi}[1-f(\xi)] d \xi\right] 0 \leqq t<s \leqq 1 .
\end{array}\right.
$$

Therefore, by using Eqs. (20) and (21), the following approximate formulas for calculating the minimum eigenvalue $V_{c}^{2}$ are derived:

First approximate formula (in the case of $m=1$ ):

$$
\begin{gathered}
T_{2}=\frac{1}{2} \int_{0}^{1} \int_{0}^{t} \frac{s^{3}}{t}[1-f(s)][1-f(t)] d s d t, \\
V_{c} \simeq \frac{1}{\left(T_{2}\right)^{1 / 4}} .
\end{gathered}
$$

Second approximate formula (in the case of $m=2$ ):

$$
\begin{gathered}
T_{4}=\frac{1}{8} \int_{0}^{1} \int_{0}^{t} t s[1-f(t)][1-f(s)] \\
\times\left[\frac{1}{t s} \int_{0}^{s} \xi^{3}[1-f(\xi)] d \xi\right. \\
\left.+\frac{s}{t} \int_{s}^{t} \xi[1-f(\xi)] d \xi+t s \int_{t}^{1} \frac{1}{\xi}[1-f(\xi)] d \xi\right]^{2} d s d t \\
V_{c} \simeq \frac{1}{\left(T_{4}\right)^{1 / 8}} .
\end{gathered}
$$

\section{B. Approximate solution by means of the approximated eigenfunction}

Let us suppose a general continuous function $g(t)$ which is expanded by the orthonormal set of eigenfunctions $\psi_{i}(t)$ as

$$
g(t)=\sum_{i=1}^{\infty} c_{i} \psi_{i}(t)
$$

where $c_{i}$ is the expansion coefficient. By using Eq. (19), the following equations are obtained:

$$
\begin{aligned}
\phi_{1}^{(n)}(t) & \equiv \int_{a}^{b} K^{n}(t, s) g(s) d s=\sum_{i=1}^{\infty} \frac{c_{i}}{\lambda_{i}^{n}} \psi_{i}(t), \\
P_{1}^{(n)} & \equiv \int_{a}^{b} g(t) \phi_{1}^{(n)}(t) d t=\sum_{i=1}^{\infty} \frac{c_{i}^{2}}{\lambda_{i}^{n}},
\end{aligned}
$$




$$
Q_{1}^{(n)} \equiv \int_{a}^{b}\left[\phi_{1}^{(n)}(t)\right]^{2} d t=\sum_{i=1}^{\infty} \frac{c_{i}^{2}}{\lambda_{i}^{2 n}} .
$$

If $g(t)$ is close enough to $\psi_{1}(t)$, the first coefficient $c_{1}$ is much larger than the other coefficients $c_{2}, c_{3}, \ldots$, i.e.,

$$
c_{1} \gg c_{2}, c_{3}, \ldots
$$

Therefore, the right-hand sides of Eqs. (29) and (30) can be approximated to be $c_{1}^{2} / \lambda_{1}^{n}$ and $c_{1}^{2} / \lambda_{1}^{2 n}$, respectively, with more accuracy than Eq. (21). Thus, the following approximate formula is obtained:

$$
\lambda_{1} \simeq\left(P_{1}^{(n)} / Q_{1}^{(n)}\right)^{1 / n}
$$

Furthermore, since the first eigenfunction $\psi_{1}(t)$ can also be approximated by $\phi_{1}^{(n)}(t)$ according to the inequality (31), the accuracy of Eq. (32) can be increased by using $\phi_{1}^{(n)}(t)$ defined by Eq. (28) instead of $g(t)$. Iterating the above process, the following successive approximate formulas are obtained:

$$
\begin{gathered}
\phi_{\nu}^{(n)}(t) \equiv \int_{a}^{b} K^{n}(t, s) \phi_{\nu-1}^{(n)}(s) d s=\sum_{i=1}^{\infty} \frac{c_{i}}{\left(\lambda_{i}^{n}\right)^{\nu}} \psi_{i}(t), \\
P_{\nu}^{(n)} \equiv \int_{a}^{b} \phi_{\nu-1}^{(n)}(t) \phi_{\nu}^{(n)}(t) d t=\sum_{i=1}^{\infty} \frac{c_{i}^{2}}{\left(\lambda_{i}^{n}\right)^{2 \nu-1}}, \\
Q_{\nu}^{(n)} \equiv \int_{a}^{b}\left[\phi_{\nu}^{(n)}(t)\right]^{2} d t=\sum_{i=1}^{\infty} \frac{c_{i}^{2}}{\left(\lambda_{i}^{n}\right)^{2 \nu}} \\
\lambda_{1} \simeq\left(P_{\nu}^{(n)} / Q_{\nu}^{(n)}\right)^{1 / n}
\end{gathered}
$$

where $\phi_{0}^{(n)}(t)=g(t)$ and $\nu$ is the number of order in the successive approximation.

By putting $\lambda_{1}=V_{c}^{2}, a=0, b=1$, and $n=1$, substitution of Eq. (14) into Eqs. (33)-(36) gives the third approximate formula for $\mathrm{TE}_{01}$ mode cutoff as follows:

Third approximate formula:

$$
\begin{array}{r}
\phi_{\nu}(t)=\frac{1}{2} \sqrt{t[1-f(t)]}\left(\frac{1}{t} \int_{0}^{t} s \sqrt{s[1-f(s)]} \phi_{\nu-1}(s) d s\right. \\
\left.+t \int_{t}^{1} \frac{1}{s} \sqrt{s[1-f(s)]} \phi_{\nu-1}(s) d s\right) \\
V_{c}=\left(\int_{0}^{1} \phi_{\nu-1}(t) \phi_{\nu}(t) d t / \int_{0}^{1}\left|\phi_{\nu}(t)\right|^{2} d t\right)^{1 / 2}
\end{array}
$$

where $n$ is put equal to 1 in order to reduce the dimension of multiple integration. According to Eq. (13), the product of $\sqrt{t[1-f(t)]}$ and any continuous function that satisfies the boundary conditions Eqs. (3) and (11) is available for the initially approximated eigenfunction $\phi_{0}^{(1)}(t)[=g(t)]$. For example, $\sqrt{t[1-f(t)]}\left(3 t-2 t^{2}\right), \sqrt{t[1-f(t)]} J_{1}(\kappa t) \quad(\kappa$ $=2.40482556)$, etc., satisfy these conditions.

\section{NUMERICAL RESULTS}

\section{A. Results by means of the first and second approximate formulas}

Since the first and second approximate formulas include no field scalar function $R(\rho)$, the $\mathrm{TE}_{01}$ mode cutoff can be arithmetically calculated for several simple index profile expressions. By substituting $f(\rho)=\rho^{\alpha}$ into Eqs. (24) and (26), the cutoff frequency of the $\mathrm{TE}_{01}$ mode of an $\alpha$ power law fiber
TABLE I. Cutoff $V_{c}$ of the $T E_{01}$ mode of a parabolic and step-index profile by means of the first and second approximate formulas.

\begin{tabular}{llll}
\hline \hline & $\begin{array}{c}V_{c} \text { by first } \\
\text { approx. formula }\end{array}$ & $\begin{array}{c}V_{c} \text { by second } \\
\text { approx. formula }\end{array}$ & Exact value \\
\hline 2 & $\begin{array}{c}3.4641 \\
(\text { error } 1.5 \%)\end{array}$ & $\begin{array}{c}3.5169 \\
(\text { error } 0.03 \%)\end{array}$ & $3.518^{\mathrm{a}}$ \\
$\infty$ & $\begin{array}{c}2.3784 \\
(\text { error } 1.1 \%)\end{array}$ & $\begin{array}{c}2.4044 \\
(\text { error } 0.02 \%)\end{array}$ & 2.40482556 \\
\hline
\end{tabular}

a The result of Refs. 4 and 7 was used as the exact value.

is obtained as

$$
V_{c} \simeq\left(\frac{1}{32}+\frac{1}{8} \frac{1}{(\alpha+2)}-\frac{1}{4} \frac{1}{(\alpha+4)}-\frac{1}{2} \frac{1}{(\alpha+4)^{2}}\right)^{-1 / 4}
$$

for the first approximation and

$$
\begin{aligned}
V_{c} \simeq & \frac{11}{3 \times 2^{12}}+\frac{5}{2^{7}} \frac{1}{(\alpha+2)}-\frac{343}{3 \times 2^{9}} \frac{1}{(\alpha+4)} \\
& -\frac{3^{3}}{5 \times 2^{6}} \frac{1}{(\alpha+6)}-\frac{13}{3 \times 2^{7}} \frac{1}{(\alpha+8)} \\
& +\frac{3^{5}}{5 \times 2^{7}} \frac{1}{(3 \alpha+8)}-\frac{3}{2^{6}} \frac{1}{(\alpha+4)^{2}}+\frac{1}{2^{4}} \frac{1}{(\alpha+4)^{3}} \\
+ & \left.\frac{1}{2^{4}} \frac{1}{(\alpha+4)^{4}}-\frac{3^{4}}{2^{6}} \frac{1}{(3 \alpha+8)^{2}}-\frac{5}{3 \times 2^{6}} \frac{1}{(\alpha+8)^{2}}\right)^{-1 / 8}
\end{aligned}
$$

for the second approximation, respectively. In order to exhibit the accuracy, comparison with the exact values for the case of $\alpha=2$ and $\alpha=\infty$ is shown in Table I. The errors of cutoff $V_{c}$ calculated by the first and second approximate

\begin{tabular}{|c|c|c|c|c|}
\hline \multirow[b]{2}{*}{$\alpha$} & \multirow[b]{2}{*}{$v$} & \multicolumn{2}{|c|}{$V_{c}$ by third approx. formula } & \multirow[b]{2}{*}{ Exact value } \\
\hline & & $\mathrm{I}^{\mathrm{a}}$ & $\mathrm{II}^{\mathrm{b}}$ & \\
\hline \multirow{3}{*}{2} & 1 & $3.5203777_{9}$ & $3.5223799_{7}$ & \multirow{3}{*}{$3.518^{c}$} \\
\hline & 2 & $3.5181549_{6}$ & $3.5182556_{2}$ & \\
\hline & 3 & $3.5180552_{0}$ & $3.5180602_{1}$ & \\
\hline \multirow{3}{*}{$\infty$} & 1 & $2.4049497_{2}$ & $2.4048256_{0}$ & \multirow{3}{*}{2.40482556} \\
\hline & 2 & 2.40482979 & $2.4048255_{8}$ & \\
\hline & 3 & $2.4048257_{3}$ & $2.4048255_{8}$ & \\
\hline
\end{tabular}
formulas Eqs. (39) and (40) are estimated to be within $2 \%$ and $0.05 \%$, respectively.

\section{B. Results by means of the third approximate formula}

Although the second approximate formula has relatively high accuracy, this formula requires much time for computation because it includes a triple integral. On the other hand, a set of third approximate formulas Eqs. (37) and (38) includes only double integrals. Therefore, third approximate formulas are suited for numerical calculations. The comparison of results calculated by using Eqs. (37) and (38) with the exact values for the case of $\alpha=2$ and $\alpha=\infty$ is shown in Table II. In this calculation, the functions $\sqrt{t[1-f(t)]}\left(3 t-2 t^{2}\right)$ and

TABLE II. Cutoff $V_{c}$ of the $T E_{01}$ mode of a parabolic and step-index profile by means of the third approximate formula.

II: $\phi_{0}(t)=\sqrt{l] 1-[(t)]}\left(3 t-2 t^{2}\right)$.

b II: $\phi_{0}(t)=\sqrt{t[1-f(t)]} J_{1}(\kappa t)(\kappa=2.40482556) ; \nu$ is the number of order in the successive approximation.

c The result of Refs. 4 and 7 was used as the exact value. 


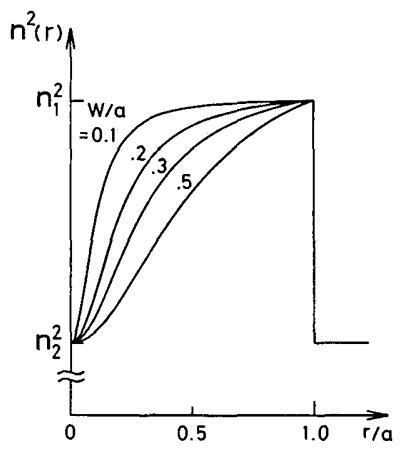

$\sqrt{t[1-f(t)]} J_{1}(\kappa t)(\kappa=2.40482556)$ were used as the initial approximated eigenfunction $\phi_{0}^{(1)}(t)$. The computation time was about 1.7 seconds per one step of successive approximation by using a middle-scale computer (FACOM 230-38S). From Table II, we see that the error of the first-order approximation is less than $0.2 \%$ and that the accuracy is improved by one order of magnitude by means of successive approximation.

The effect of a center dip on the cutoff frequency was investigated by using the second-order approximation of Eqs. (37) and (38). Thus far, the index profile function of the center dip has been expressed by the inverse $\alpha$-power-law function. ${ }^{10}$ But the parameter $\alpha$ in this expression does not correspond directly to the width of the center dip. In this paper, the index profile function of the center dip is expressed by a Lorentzian function,

$$
f(\rho)=\frac{(W / a)^{2}\left(1-\rho^{2}\right)}{(W / a)^{2}+\rho^{2}},
$$

where $a$ is the core radius and $W$ is the width of the center dip. This profile is illustrated in Fig. 1. As for the initial approximated eigenfunction, $\phi_{0}^{(1)}(t)=\sqrt{t[1-f(t)]}\left(3 t-2 t^{2}\right)$ was used. The result is shown in Fig. 2. The cutoff frequency $V_{c}$ can be approximated by $2.4048+1.86 W^{2} / a^{2}$ when the dip width $W$ is small $(W / a<0.15$ ) and by $2.3445+0.6065 W / a$ when the dip width is large $(W / a>0.25)$.

\section{CONCLUSION}

In this paper, three types of approximate formulas for calculating the $\mathrm{TE}_{01}$ mode cutoff of a radially inhomogeneous optical fiber were derived.

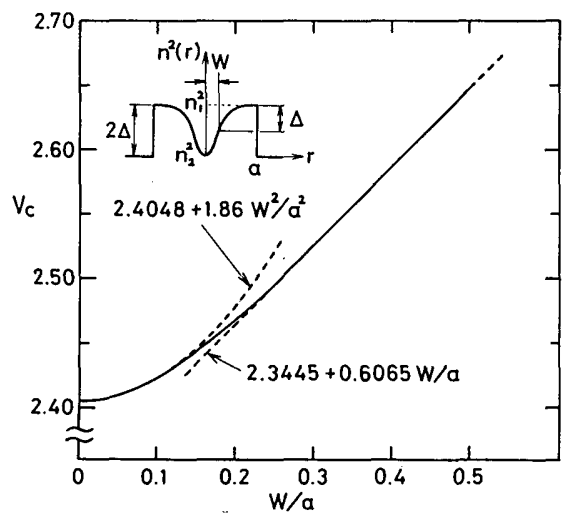

FIG. 2. Cutoff $V_{c}$ of the $T E_{01}$ mode of the refractive index profile with Lorentzian-form center dip.

TABLE III. Summary of the features of the approximate formulas.

Features

First approximate formula

Second approximate formula

Third approximate formula
This includes only the refractive index profile function.

This can be used for an arithmetic calculation if the index profile function is expressed by a simple formula.

The error is within about $2.0 \%$ and this is not of satisfactory accuracy.

The calculated value is always smaller than the exact value.

This includes only the refractive index profile function.

This requires much time for arithmetic and numerical calculations because of the triple integral.

The error is within about $0.05 \%$ and this is of practical use.

The calculated value is always smaller than the exact value.

This requires the initial approximated eigenfunction.

The accuracy can be improved by one order of magnitude by means of successive approximation, whatever function is used as the initial approximated eigenfunction.

This is suitable for numerical calculation because this includes only double integrals.

The first approximate formula can be used as a simple formula since it includes only the index profile function, but the error is relatively large (within $2 \%$ ). The second approximate formula has higher accuracy (within $0.05 \%$ ), but it is not suitable for arithmetical and numerical calculation because it includes a triple integral. The third approximate formula is suitable for numerical calculation because it includes only double integrals. The error of this formula can be reduced up to the limit of numerical integration owing to successive approximations. Therefore, the third approximate formula is best for practical use and gives the least error.

In this paper, we investigated the cutoff frequency of only the $\mathrm{TE}_{01}$ mode. However, this method can be applied to the $\mathrm{TM}_{01}$ and $\mathrm{HE}_{21}$ modes. Furthermore, $V-b$ curve (dispersion curve) can be obtained by calculating the $V$ value versus a given value of $w$ by means of Eq. (9). These calculations are planned to be presented later.

The features of these three formulas are summarized in Table III.

\section{ACKNOWLEDGMENT}

The authors express their sincere thanks to Professor Y. Suematsu and Associate Professor K. Furuya for their valuable discussions of this work.

1J. Sakai and T. Kimura, "Large-core broadband optical fibers," Opt. Lett. 1, 169-171 (1977)

${ }^{2}$ K. Iga, Y. Kokubun, and H. Ono, "A design and experiment for 
group-delay-difference-free dual mode optical fibers," paper of TGOQE, IECE Japan, No. OQE78-36 (1978).

${ }^{3}$ E. Snitzer, "Cylindrical dielectric waveguide modes," J. Opt. Soc. Am. 51, 491-498 (1961).

${ }^{4}$ T. I. Lukowsky and F. P. Kapron, "Parabolic fiber cutoffs: A comparison of theories," J. Opt. Soc. Am. 67, 1185-1187 (1977).

5S. Kawakami, S. Nishida, and M. Sumi, "Transmission characteristics of W-type optical fibers," Proc. IEE 123, 586-590 (1976).

${ }^{6}$ A. Kumar, R. Chanda, R. A. Sammut, and A. K. Ghatak, "Cutoff frequencies of a parabolic-core W-type fiber," Electron. Lett. 14, . 676-678 (1978).
${ }^{7}$ W. A. Gambling, P. N. Payne, and H. Matsumura, "Cutoff frequency in radially inhomogeneous single-mode fibre," Electron. Lett. 13, 139-140 (1977).

${ }^{8} \mathrm{~K}$. Hotate and T. Okoshi, "A formula giving cutoff frequencies of modes in an optical fiber having arbitrary refractive-index profile," Trans. IECE Jpn. E62, 1-6 (1979).

${ }^{9} \mathrm{~J}$. Kondo, Integral Equations and their Applications (Corona, Tokyo, 1959) (in Japanese).

${ }^{10}$ W. A. Gambling, P. N. Payne, and H. Matsumura, "Effect of dip in the refractive index on the cutoff frequency of a single-mode fiber," Electron. Lett. 13, 174-175 (1977). 